\title{
Drag Reduction Mechanism of Viscoelastic Slick-Water Fracturing Fluid in Tortuous and Rough Fractures
}

\author{
Zhiyu Liu, ${ }^{1}$ Fan Fan, ${ }^{2}$ Donghang Zhang, ${ }^{3}$ Yang Li, ${ }^{4}$ Yuan Li, ${ }^{1}$ Kai Yang, ${ }^{1}$ Hongkui Ge ${ }^{1},{ }^{1}$ \\ and Fujian Zhou iD ${ }^{1}$ \\ ${ }^{1}$ The Unconventional Natural Gas Institute, China University of Petroleum, Beijing, China \\ ${ }^{2}$ Beijing Utilities Engineering Design and Supervision Co., Ltd, Beijing, China \\ ${ }^{3}$ No.1 Gas Production Plant, Sinopec Southwest Oil \& Gas Company, Deyang, Sichuan 618000, China \\ ${ }^{4}$ Deli Zhongcheng Petroleum Engineering Co., Ltd, Xinjiang, China
}

Correspondence should be addressed to Fujian Zhou; zhoufj@cup.edu.cn

Received 1 September 2021; Accepted 11 November 2021; Published 6 December 2021

Academic Editor: Amer Syed

Copyright (c) 2021 Zhiyu Liu et al. This is an open access article distributed under the Creative Commons Attribution License, which permits unrestricted use, distribution, and reproduction in any medium, provided the original work is properly cited.

Slick-water can effectively reduce the flow drag of fracturing fluid. Many studies have focused on the drag reduction performance of slick-water in wellbore and perforation, but there has been little research on drag reduction characteristics in fracture flow. In this paper, a new visualization experiment system is used to simulate real fracture. The fracture surface is produced through actual triaxial hydraulic fracturing and is copied by a three-dimensional printer using resin material to maintain its shape feature. In comparing the experimental results, it was found that the main factors affecting drag reduction in a fracture are the relative molecular weight and the added concentration. Unlike the flow rule of the drag reducer in a pipeline, when the concentration is greater than $0.10 \%$, a negative DR effect begins to appear. The influence of molecular weight is related to the flow stage; the increasing of molecular weight causes a reduction in DR effect when the flow rate is $0.24 \mathrm{~m} / \mathrm{s}$. However, the flow rate exceeds $0.5 \mathrm{~m} / \mathrm{s}$; drag reducers with higher molecular weight demonstrate better drag reduction performance. The drag reduction mechanism analysis in fractures was obtained from visualization observations, and the flow characteristics of fluid were characterized by using tracking particles. Drag reduction effect occurs mainly on the surface of the fractures in contrast to near the centre of the flow channel. This research can provide a reference for the experimental study on drag reduction in fractures and is of great significance to the optimization and improvement of drag reducing agent.

\section{Introduction}

Slick-water fracturing is a systemic method that increases production by injecting several million gallons of water into a formation to create a fracture network [1-4]. The fracturing slick-water is injected with proppant at a high pump rate to maintain the flow channel. Slick-water is commonly used in large-scale volume fracturing for its low flow drag, which is an advantage in creating more fractures and complex fracture networks to maximise the initial production rate, as consistently observed in the field [5-7]. Accurate estimation of fracturing flow drag is critical to fracturing design, and drag reduction performance is one of the most important technical indicator of slick-water fracturing fluid.
Drag reduction phenomenon is also called the "Toms" phenomenon. Only a small amount of additives can produce a significant pressure drop in turbulent flow, known as the drag reduction effect (DR effect) $[8,9]$. There are three main types of drag reduction agents (bubbles, polymers, and surfactants) often used in slick-water. Polymer as the most commonly used type of slick-water additive is a kind of water-based fracturing fluid mixed with long-chain polymers such as polyacrylamide, polyethylene oxide, and guar derivatives [10-14]. Since the DR effect was discovered, many applications have been produced. However, the exact mechanism of drag reduction is still not reach a unified understanding and be widely accepted [15-17]. Previous drag reduction mechanism research has focused mostly on 
wellbore flow drag. According to the results of previous study, the drag reduction performance of slick-water is dependent on the balance between the viscous modulus and the elastic modulus and is determined by the microstructure of the drag reducer. As a decisive factor in fracturing design, fracture flow drag requires research of flow patterns and drag reduction characteristics [18-20]. Although research on the pipeline flow has provided us with a foundation, the flow patterns and characteristics in the fractures need further thorough research.

The related research on the drag reduction mechanism of fluid in reservoirs did not concentrate on fractures but focused on microchannels or the microfractures in reservoirs. Some researchers advocated using the microfluidic chip to simulate the drag reduction flow in microfractures [21-23]. On contrast to the pipeline flow experiment, the fluid flow in microfracture is under laminar flow regime. The same drag reduction agents do not decrease the injection pressure but increased more than 50\% [24]. Then, other researchers represented by Barrat further demonstrated that adsorption properties of surfactants are the main reason leading to drag reduction effect. They believe that the surfactants adsorbed on the wall of reservoir microchannels weaken the interaction between water molecules and therefore reduce the flow drag [25-28]. Generally, previous research studies the drag reduction mechanism in microscale, and the drag reduction agents used in the research are mainly surfactants. Few studies have investigated the flow characteristics of large-scale artificial fractures. What is more, most related research does not consider the roughness and tortuosity of the fractures. The representative results were published by Fengchen and Kawaguchi; experiments were executed using PIV method to test the quantitative characteristics of vortex structures and turbulent events in two-dimensional channel made of transparent acrylic resin [29]. The literature analyses the changes of turbulence structure but ignores the influence of real fracture parameters. So the drag reduction flow in large-scale artificial fractures has not been sufficiently studied through indoor experimental simulation. The drag reduction mechanism in fracture flow still not clear and cannot be universalized with the mechanism in pipeline flow.

In this study, a self-developed indoor drag testing cycle system considering fracture tortuous and roughness was constructed to test the flow drag in both pipeline and fracture. Essential experiments have been conducted to investigate the factors influencing the DR effect in fractures including concentration, temperature, molecular weight, and electrical characteristics. The experimental research in pipeline is mainly used as a reference to explore the drag reduction characteristic in fractures. As the DR mechanism has not been extended to fractures, considering the experimental differences between pipelines and fractures, a visualization fracture model is added. The flow characteristics of fluid are characterized by using tracking particles. Although the experiment is designed as complete as possible, there remain some limitations in this study. The accuracy of the test equipment can be guaranteed and is sufficient for drag reduction rate tests. However, the experimental conditions are not sufficient to confirm the value of $R_{e}$ in fractures. Thus, further discussion on the relation between $R_{e}$ and drag reduction characteristic is not presented.

\section{Methodology}

2.1. Research Equipment. The drag reduction performance of slick-water can be analysed using an indoor visualization drag testing cycle system [30]. A diagram of the experimental apparatus is shown in Figure 1. The testing system consists of five parts: (A) a liquid supply system including three plastic buckets; the one in the middle is the effluent water bucket and is the largest, with a volume of $70 \mathrm{~L}$. Water and solution buckets with a volume of $50 \mathrm{~L}$ are on each side. The power output depends on a screw pump; its maximum pumping rate is $2.5 \mathrm{~m}^{3} / \mathrm{h}$. To better simulate flow conditions in the field, the solution buckets were equipped with a heater to test different fluid temperatures; (B) the pipeline testing system consisting of three pipelines. The pipeline diameters are $6 \mathrm{~mm}, 8 \mathrm{~mm}$, and $10 \mathrm{~mm}$; all pipelines are $3 \mathrm{~m}$ in length. With ball valves on both ends and a differential pressure transducer $(0-0.5 \mathrm{MPa}, \pm 0.02 \%)$ in the centre, to minimise the additional shear effect from the corners connected to each pipeline, pressure taps were set $0.25 \mathrm{~m}$ from each end to measure the $2.5 \mathrm{~m}$ pressure drop; (C) a visualization fracture testing system with a stainless steel frame $(360 \mathrm{~mm}$ long, $140 \mathrm{~mm}$ high) with adjustable width for fitting the surface and visualization fracture model; (D) a data acquisition system consisting of a computer and a control system to control the equipment and collect flow rate, temperature, and pressure data; (E) a pressure sensing system with a differential pressure transducer $(0-100 \mathrm{kPa}, \pm 0.001 \%)$ to test the pressure drop between visual fractures with taps set $30 \mathrm{~mm}$ from each end to measure the $300 \mathrm{~mm}$ pressure drop. The system was designed to simultaneously measure pipeline and fracture flow drag and to precisely compare their differences in the same flow conditions.

2.2. Visualization Fracture Model. The visualization fracture model is showed in Figure 2, and the main body of the model is ASTM304 steel frame. Truly simulated fracture surface is made of resin material. There is an injection interface on one side and a flow out interface on the other side. Moreover, there is an injection connector on one side and a flow out connector on the other side. In order to introduce the model production process in specifically, the equipment manufacturing process is divided into three parts. The tortuous and rough fracture surface was created through largescale actual triaxial hydraulic fracturing, as shown in Figure 3, and the rock size was $30 \mathrm{~cm}^{3}$. A surface scanner was used to accurately describe the roughness and tortuosity of the fracture, which was copied by a three-dimensional printer using resin to maintain its clarity. The copied surface was assembled into a circumjacent and airtight stainless steel frame, and the size of the frame is $360 \mathrm{~mm}$ long and $140 \mathrm{~mm}$ high. The distance between this two surface can be adjusted to the fracture width from 1 to $8 \mathrm{~mm}$. As shown in Figure 4, the visualization fracture model is connected with the loop testing system. A pressure transducer was installed $(0-100 \mathrm{kPa}$, 


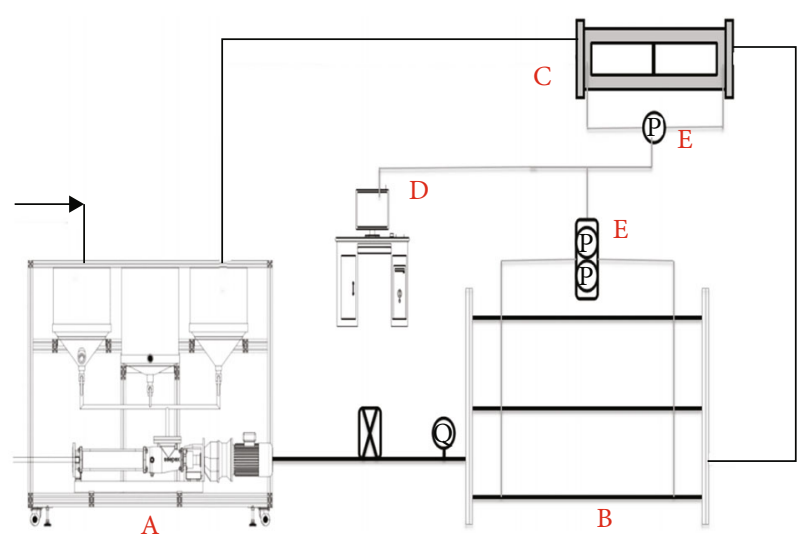

FIGURE 1: Diagram of experimental apparatus: (a) liquid supply system; (b) pipeline test system; (c) visualization fracture model; (d) data acquisition system; (e) pressure sensing system.

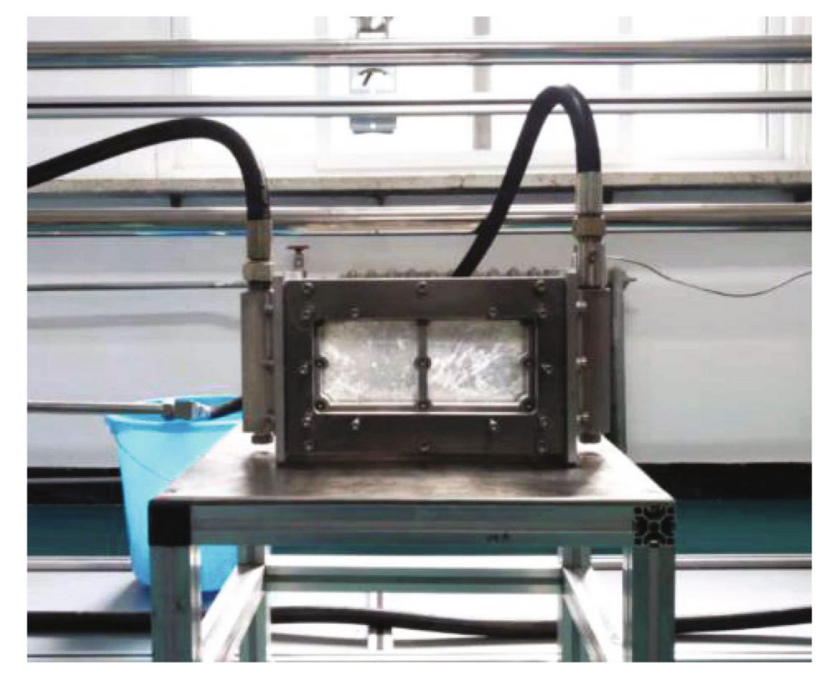

FIGURE 2: Visualization fracture model.
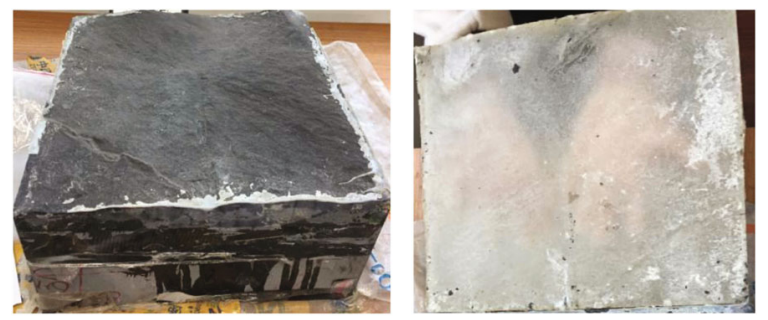

FIGURE 3: Large-scale actual triaxial hydraulic fracturing surface and copied fractures.

$\pm 0.01 \%)$ on each connector to monitor the changes of flow pressure, and the two pressure spots were set at a distance of $300 \mathrm{~mm}$.

2.3. Selected Drag Reducer. Polyacrylamide polymer drag reducers are commonly used in hydraulic fracturing and can be generally categorised as cationic, anionic, or nonionic, according to their electrical characteristics after hydrolysis. Cationic drag reducers are relatively expensive.

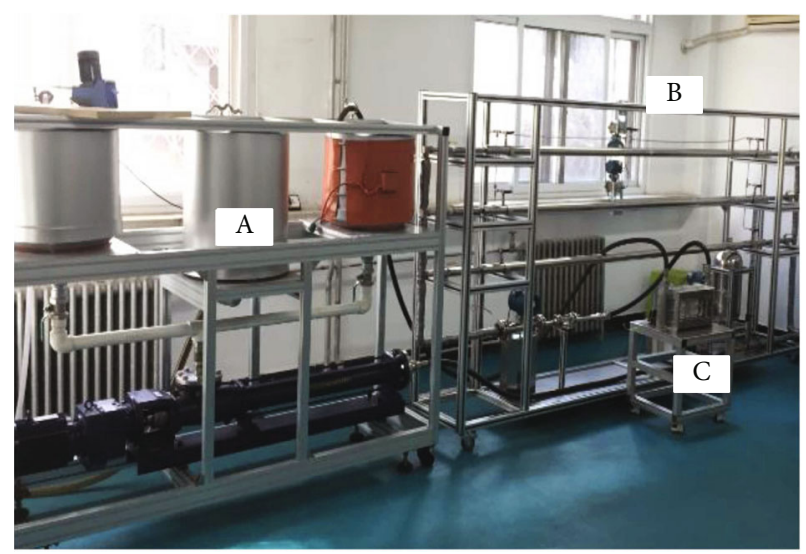

Figure 4: Indoor visualization drag testing cycle system: (a) liquid supply system; (b) pipeline test system; (c) visualization fracture model.

Nonionic drag reducers require an amount approximately one order of magnitude greater to be added than cationic and anionic reducers for the same drag reduction effect. Thus, anionic drag reducers are usually used for fracturing [31]. The drag reducer used in this study is an emulsion form and water-soluble drag reducer and is synthesized from a mixture of acrylamide (AM), acrylic acid (AA), 2-acrylamido-2-methylpropanesulfonic acid (AMPS), and butyl acrylate (BA) in aqueous solution by the polymerization process. It is manufactured by Shengli Chemical Co., Ltd., commercially known as DR800. It is an anionic polyacrylamide with a high molecular weight of $8 \times 10^{6}$ and several long side groups. DR800 was mixed with tap water to synthesize slick-water, a commonly used fracturing fluid in unconventional reservoir development. The relationship between slick-water viscosity and drag reducer dosage was measured using a Ubbelohde viscometer at $25^{\circ} \mathrm{C}$; the results are shown in Figure 5.

2.4. Data Processing. Drag reduction efficiency (DR\%) is calculated as

$$
\mathrm{DR} \%=\frac{C_{f D}-C_{f}}{C_{f D}} \times 100 \%,
$$

where $C_{f D}$ is the Fanning friction factor of water and $C_{f}$ is the Fanning friction factor of slick-water in the same flow conditions, defined as

$$
C_{f}=\frac{2 \tau_{w}}{\rho U_{b}^{2}}
$$

where $\tau_{w}$ is the wall shear stress, $\rho$ is the density of the solvent, and $U_{b}$ is the average flow velocity. Figure 6 shows a diagram of the fracture and pipeline.

For a fracture, Equation (2) can be deduced as

$$
C_{f}=\frac{2 \tau_{w}}{\rho U_{b}^{2}}=\frac{\Delta p H W}{\rho U_{b}^{2}(H+W) L},
$$




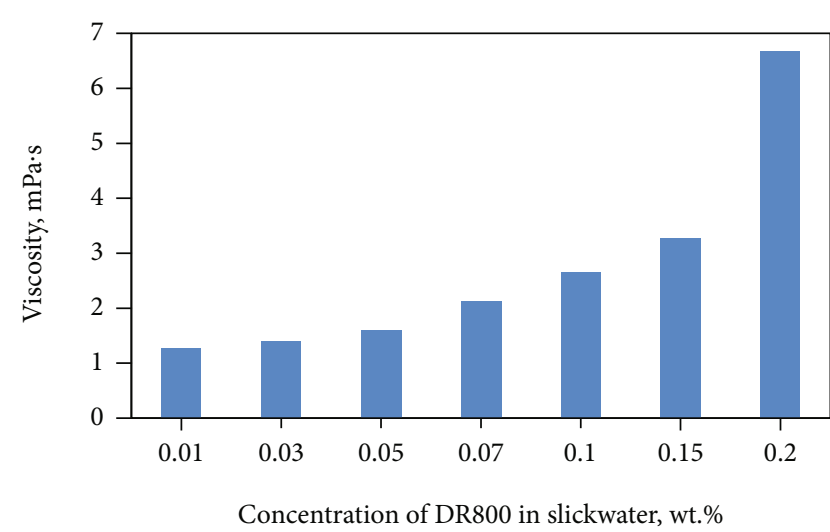

Figure 5: Relationship between slick-water viscosity and drag reducer concentration.
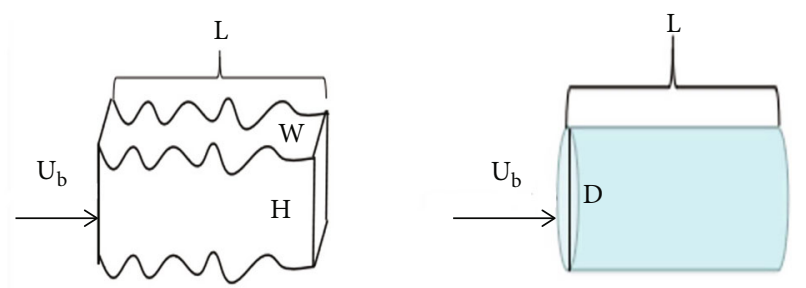

FIgURE 6: Diagram of fracture and pipeline.

where $\Delta p$ is the pressure drop over the two pressure taps, $L$ is the distance between the pressure taps, and $H$ and $W$ are the height and width of the fracture, respectively.

For a pipeline, Equation (2) can be deduced as

$$
C_{f}=\frac{2 \tau_{w}}{\rho U_{b}^{2}}=\frac{2 D \Delta P}{\rho U_{b}^{2} L},
$$

where $D$ is the inner diameter of the pipeline.

The largest polymer dosage in our experiment was 0.15 wt.\%; the density of the $0.15 \mathrm{wt} . \%$ solution was $997.043 \mathrm{~kg} / \mathrm{m}^{3}$, which is nearly the density of water. Thus, the displacement is the same. For each test section, Equation (1) can be reduced as

$$
\mathrm{DR} \%=\frac{\Delta P_{w}-\Delta P_{\mathrm{DR}}}{\Delta P_{w}} \times 100 \%,
$$

where $\Delta P_{w}$ is the pressure drop of water.

The Reynolds number $R_{e}$ is defined as

$$
R_{e}=\frac{\rho U_{b} R}{\mu},
$$

where $\mu$ is the fluid viscosity and $R$ is the hydraulic radius.

For a fracture, $R_{f}=H W / 2(H+W) ; R_{e}$ can be deduced as

$$
R_{e}=\frac{\rho U_{b} H W}{2 \mu(H+W)}
$$

For a pipeline, $R_{p}=D / 2 ; R_{e}$ can be deduced as

$$
R_{e}=\frac{\rho U_{b} D}{2 \mu} .
$$

In pipeline flow, the Reynolds number is an important parameter to distinguish laminar and turbulent flows but is not suitable for fractures. An explicit critical Reynolds number between laminar and turbulent flows has not been established for true complicated fracture flow.

2.5. Equipment Reliability. Equipment reliability was checked prior to the experiments. Considering that there are no mature empirical formulas or judgment bases for true complicated fracture flow drag, repeated experiments were performed to test the water flow drag in fractures under the same conditions. Figure 7 shows perfect repeatability, indicating high experimental device accuracy and providing strong evidence of drag reduction experiment reliability.

\section{Results and Discussion}

3.1. DR Performance. Generally, the DR concentration is approximately $0.05 \mathrm{wt} . \%$ to $0.15 \mathrm{wt} . \%$ in the field, considering both performance and economy [32]. In this study, the DR\% of 0.05 wt.\% DR800 solution with the same displacement in the fractures and pipeline are presented in Figure 7. As shown in Figure 8, in the same pumping displacement conditions, the maximum drag reduction rate in the pipeline flow exceeds $70 \%$; the maximum drag reduction rate in the fracture flow does not exceed 7\%, a difference of more than ten times. Thus, at the same displacement, the drag reduction flow characteristics in a pipeline and a fracture are completely different. This is a result of different flow channel cross-sectional shapes and many other factors. However, it is certain that the pump displacement parameters cannot be used as effective variables in the comprehensive evaluation of drag reduction in pipelines and fractures.

To better observe the DR performance in fractures, the variation regularity of the drag reduction rate was analysed with different average flow rates and Reynolds numbers. The experimental results are presented in Figure 9(a). For 0.05 wt.\% DR800, the drag reduction rate increases with an increase in the average flow rate and Reynolds number. Under these two conditions, the variation regularity of the drag reduction rate is essentially the same. The actual Reynolds number of the fluid in tortuous and rough fractures is difficult to determine. It is more accurate to analyse the drag reduction performance in fractures in average flow rate conditions.

For comparison, the drag reduction performance of 0.05 wt.\% DR800 in pipeline flow was also tested. The test results are presented in Figure 9(b). In pipeline flow conditions, the average flow rate reaches $2-18 \mathrm{~m} / \mathrm{s}$, greater than ten times the average flow rate in fracture flow. In pipeline flow, the Reynolds number at the minimum average flow rate must be greater than 15000 (the critical value for dividing laminar turbulent flow is 2100). However, in fracture flow, the Reynolds number is less than 2100 , even at the 


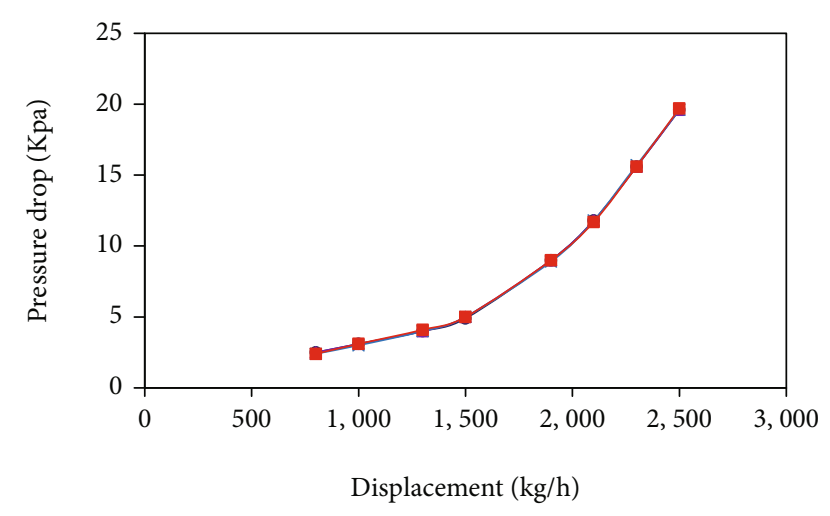

$\rightarrow$ Water fracture flow drag test $1 \#$

^ Water fracture flow drag test 2\#

$\rightarrow$ Water fracture flow drag test $3 \#$

$\rightarrow$ Water fracture flow drag test $4 \#$

FIGURE 7: Results comparison in repeated experiments.

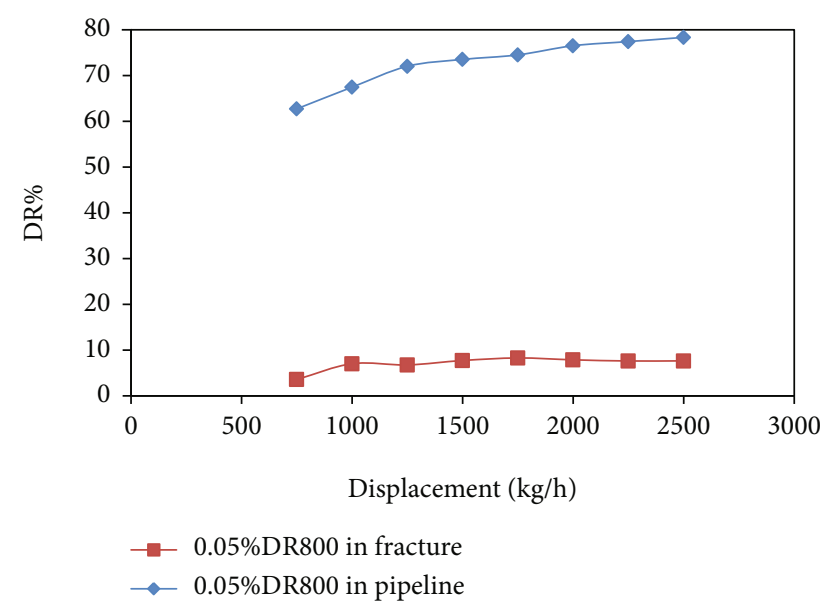

Figure 8: DR\% of 0.05 wt.\% DR800 with the same displacement in fracture and pipeline.

maximum average flow rate. Thus, the difference in the pipeline flow and fracture flow states is the main reason for the large difference in drag reduction performance. In different flow states, the drag reduction effect in pipeline flow occurs mainly in the centre of the flow channel where the turbulence is more concentrated; the drag reduction effect in fracture flow occurs mainly near the fracture surface.

Experiments were conducted to study the factors influencing DR\% in fractures. To guarantee the accuracy of the experimental results, all experiments were repeated at least twice, and the results were averaged.

\subsection{Factors Influencing the DR Effect}

3.2.1. DRA Concentration. Eight concentration groups of DR800 were tested at $25^{\circ} \mathrm{C}$ with different flow rates. Figure 10 shows the influence of the DRA concentration on the DR effect in pipelines. At a low flow rate, an increase in additive concentration leads to an increase in viscosity, which increases the relative flow resistance and the drag reduction. The DR\% of 0.01 wt.\% DR800 decreases rapidly at $6 \mathrm{~m} / \mathrm{s}$, indicating that the liquid at this concentration has the greatest shearing resistance. When the flow velocity was increased, the DR\% of 0.03 wt.\% DR800 began to decrease slowly after reaching the peak value, indicating that its DR performance had reached its limit. With an increase in the flow rate, the DR\% of 0.05 wt.\% DR800 increased gradually, indicating that deformation or failure of the molecular structure may have occurred while achieving efficient drag reduction. The DR performance of $0.20 \mathrm{wt} . \%$ DR800 indicates that high viscosity is the greatest obstacle to drag reduction at low flow rates, which was further verified by the DR performance in the fracture.

Figure 11 shows the influence of DRA concentration on the DR effect in a $3 \mathrm{~mm}$ fracture, which is different from that in a pipeline; a higher concentration increases the DR effect. At high concentrations, the DRA cannot reduce the flow drag and increases the flow resistance compared with pure water. This phenomenon is referred to as a negative DR effect. At each concentration, the flow rate has a positive effect on the DR effect and an inhibiting effect on the negative DR effect. When the concentration is greater than $0.10 \%$, a negative DR effect begins to appear. To further determine the critical concentration and to better analyse the change rule of the negative DR effect, additional high concentration drag reducer performance tests were conducted (0.09 wt.\% DR800, 0.11 wt.\% DR800, 0.13 wt.\% DR800). As shown in Figure 12, 0.09 wt.\% DR800 solution still reduces the flow drag, but 0.11 wt.\% DR800 solution produces a negative drag reduction effect. This further proves that the critical concentration is $0.1 \mathrm{wt} . \%$.

The results of experimental research on the influence of concentration indicated that drag reduction agents have the optimal concentration under relevant drag reduction flow conditions (both in pipeline flow and fracture flow). When pipeline flow is under low flow rate, concentration has an adverse effect on DR effect. But as the increases of flow rate, high concentrations of drag reducers begin to show benefit to their effect. In relation to fracture flow, increasing of drag reducer concentration causes reduction in drag reduction rate. The reason is mainly because the overall flow rate in the fracture is under lower level; high viscosity caused by concentration increases has a more significant negative impact on DR effect and leads to an increase in energy consumption, eventually caused a decrease in drag reduction effect.

3.2.2. Temperature. Five groups of $0.05 \mathrm{wt} . \%$ DR 800 were tested at different flow rates at $25^{\circ} \mathrm{C}, 35^{\circ} \mathrm{C}, 45^{\circ} \mathrm{C}, 55^{\circ} \mathrm{C}$, and $65^{\circ} \mathrm{C}$ in a circular pipeline. Within $45^{\circ} \mathrm{C}$, temperature had a positive effect on drag reduction performance, as shown in Figure 13, indicating that a viscosity decrease accompanied by a temperature increase promotes drag reduction. The DR performance at $55^{\circ} \mathrm{C}$ began to decline at $12 \mathrm{~m} / \mathrm{s}$ and rapidly declined at $65^{\circ} \mathrm{C}$, indicating that the shearing resistance ability was significantly weakened by the joint action of high flow rate and high temperature.

Five groups of $0.05 \mathrm{wt} . \%$ DR800 were tested in fracture flow at different flow rates at $25^{\circ} \mathrm{C}, 35^{\circ} \mathrm{C}, 45^{\circ} \mathrm{C}, 55^{\circ} \mathrm{C}$, and 

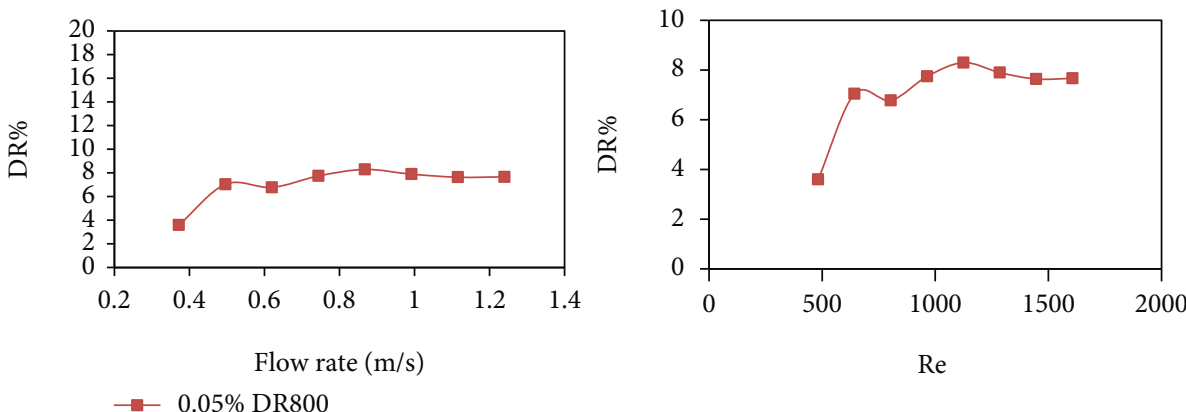

(a) Fracture flow
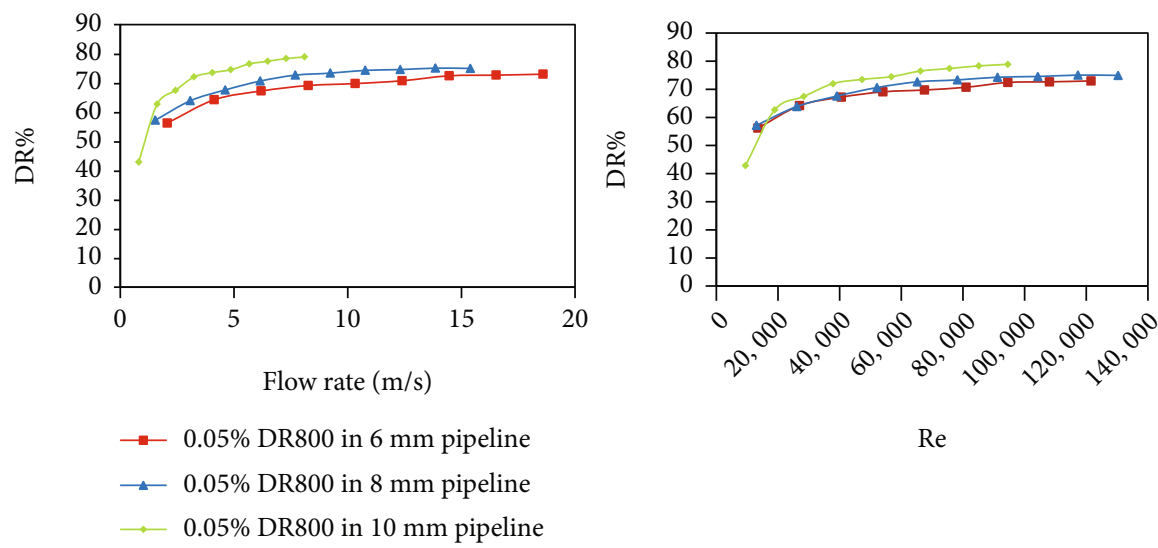

Re

(b) Pipeline flow

Figure 9: Relation of DR\% with flow rate and Reynolds number.

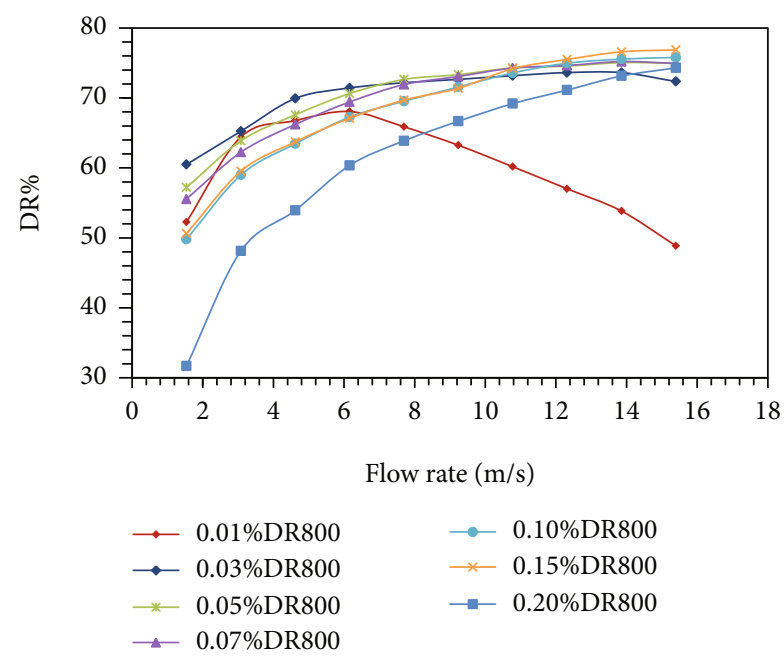

FIGURE 10: Influence of DRA concentration on DR effect in pipeline.

$65^{\circ} \mathrm{C}$. Within $45^{\circ} \mathrm{C}$, temperature has a positive effect on drag reduction performance, as shown in Figure 14, because viscosity declines with increasing temperature, which is beneficial for the DR effect. At higher temperatures, the DR effect increased at low velocities and decreased at high velocities because excessive molecular thermal motion impedes the form of the DR structure; a small shear force can influence DR\%.
3.2.3. Polymer Molecular Weight. The type of DRA was changed based on previous experiments. In this study, research was conducted on the influence of polymer molecular weight and polymer molecular structure on the DR effect as related to polymer-based drag reduction agents. Several anionic polymer DRA with different molecular weights at a given concentration were chosen for comparison and were named according to their molecular weight. DR800 was used as the reference. DR2000 and DR6000 are polymers with several long side groups, while DR1200 is a high polymer with many short side groups, as shown in Figure 15. From previous studies, it is generally acknowledged that the DR effect improves with increasing molecular weight under low flow rate conditions; short side groups are not conducive to the performance of drag reduction, and long side groups increase the drag reducing rate.

Figure 16 shows the DR\% comparison for $0.05 \mathrm{wt} . \%$ DRA in pipelines. The influence of molecular weight on $\mathrm{DR} \%$ is more obvious in the low flow rate section. At low flow rate $(1.8 \mathrm{~m} / \mathrm{s})$, the DR effect is rising with an increase in molecular weight. But when the flow rate exceeded $8.0 \mathrm{~m} / \mathrm{s}$, the DR effect was tended to the same value. In comparison, DR1200 exhibited a better DR effect than the others at high velocity, indicating that a high flow rate can alleviate the disadvantageous effects of short side groups.

Figure 17 shows that $0.05 \mathrm{wt} . \%$ DRA in a $3 \mathrm{~mm}$ fracture is different than in a pipeline. Long side groups may not have had enough space for expansion; the increasing of molecular weight causes a reduction in DR effect. With 


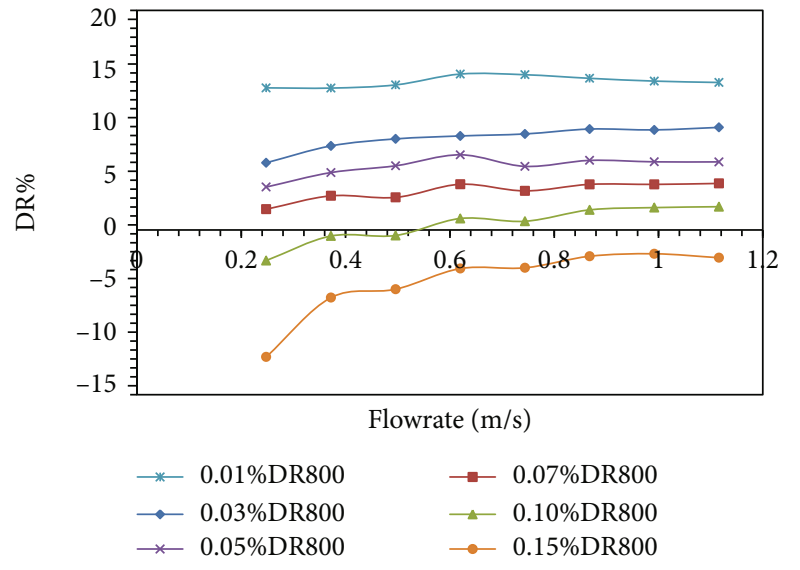

Figure 11: Influence of DRA concentration on DR effect in fracture.

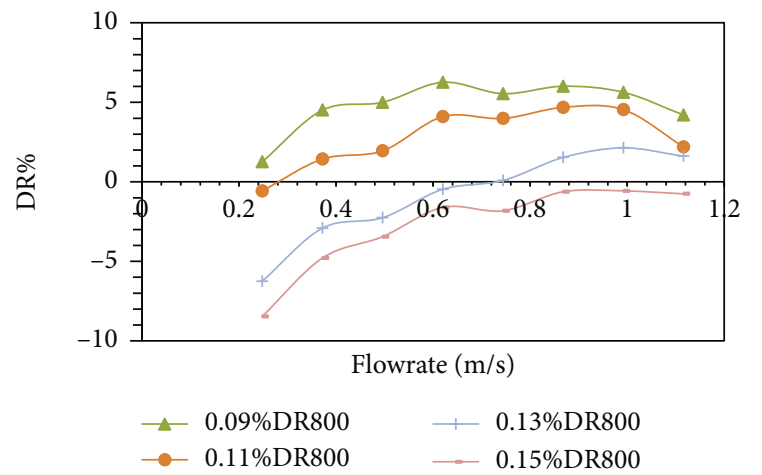

FIGURE 12: Influence of high DRA concentration on negative DR effect.

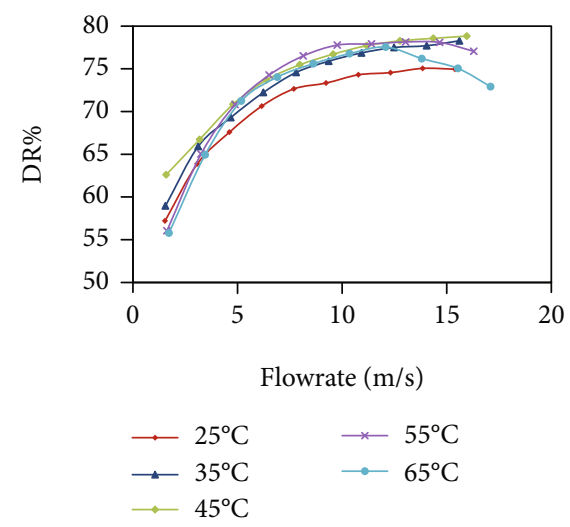

Figure 13: Relation of DR\% and flow rate at different temperatures in pipelines with $0.05 \mathrm{wt} . \%$ DR800.

increasing of flow rate, the positive influence of molecular weight becomes apparent. As the influence of the tortuously and roughness of the fracture surface, the rule of polymer molecular weight affecting drag reduction performance has changed. When the flow rate is $0.24 \mathrm{~m} / \mathrm{s}$, the increase in molecular weight is not conducive to drag reduction performance in fracture flow. However, when the flow rate exceeds $0.5 \mathrm{~m} / \mathrm{s}$, drag reducers with higher molecular weight demon-

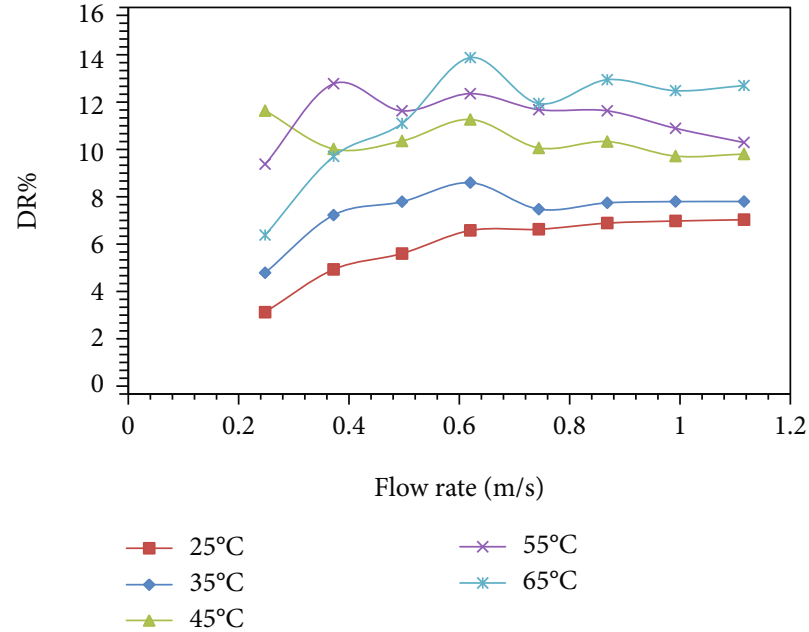

FIGURE 14: Relation of DR\% and flow rate at different temperatures in fracture for $0.05 \%$ DR800.

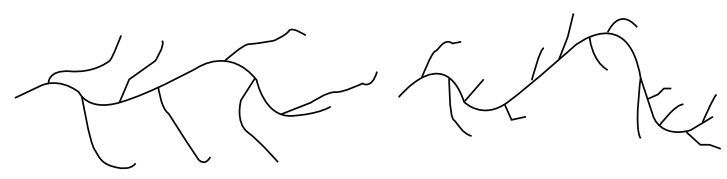

FIgURE 15: Two molecular DRA structures.

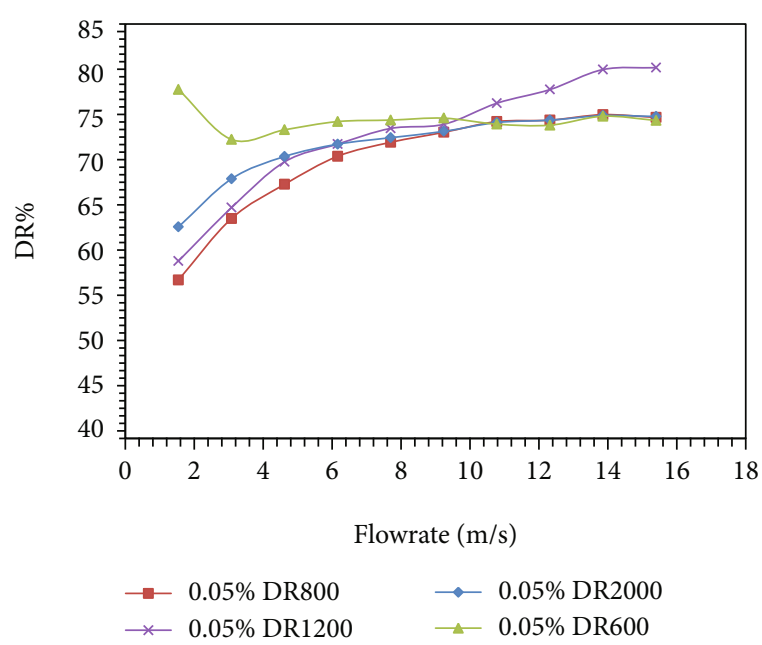

Figure 16: Comparison of $0.05 \%$ DRA in $8 \mathrm{~mm}$ pipeline.

strate better drag reduction performance. As stated above, the molecular weight has significant difference effects on drag reduction performance in pipelines and fractures. DR1200 exhibited a negative DR effect, indicating that the short side groups decrease the DR effect, especially in fracture flow.

3.2.4. Polymer Electrical Characteristics. Commonly used DRA in slick-water fracturing can be generally categorised as cationic, anionic, or nonionic according to their electrical characteristics after hydrolysis. Cationic, anionic, and nonionic DRA with the same molecular weight and molecular structure were chosen to study the influence of electrical 


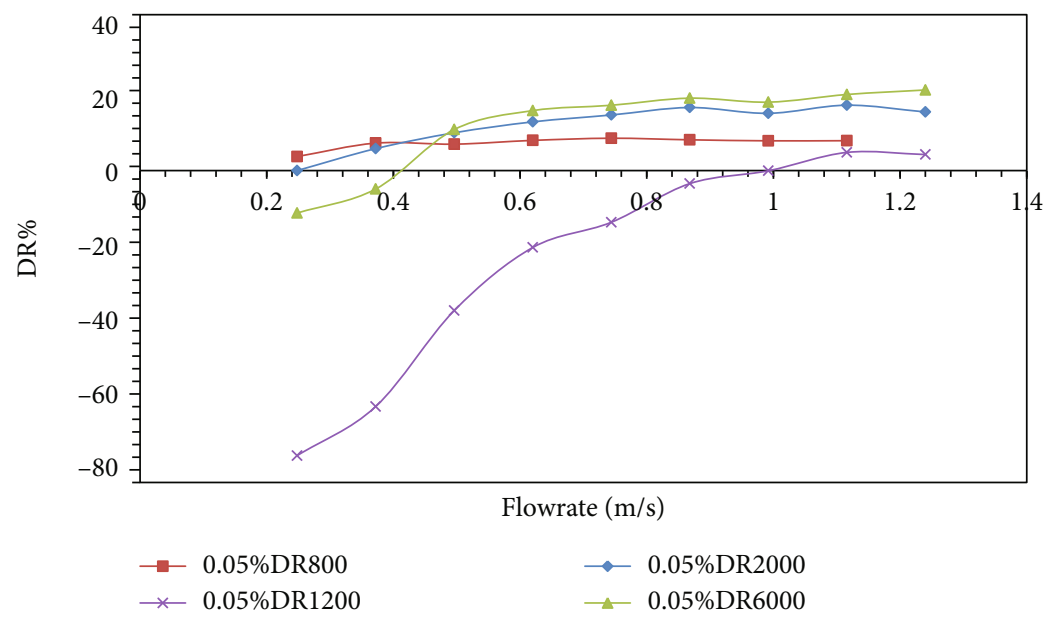

FIgURe 17: Comparison of $0.05 \%$ DRA in $3 \mathrm{~mm}$ fracture.

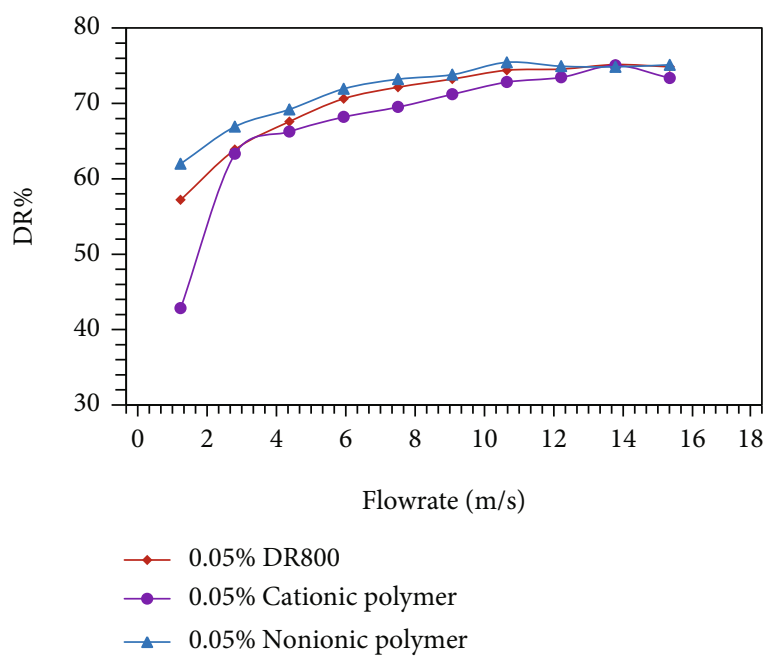

Figure 18: Results for three DRA types in $8 \mathrm{~mm}$ pipeline.

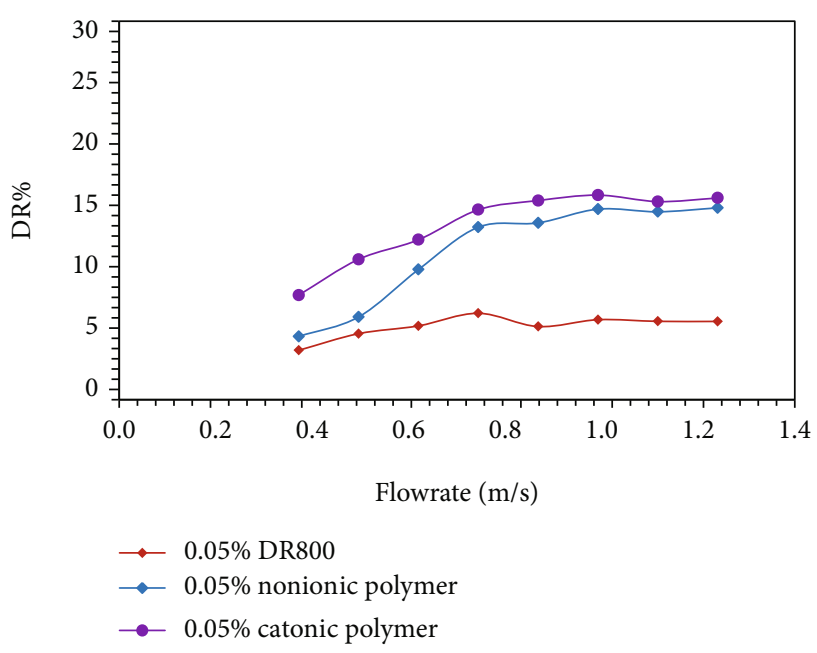

FIgURE 19: Results for three DRA types in $3 \mathrm{~mm}$ fracture. characteristics on the DR effect. Figure 18 shows the DR\% results for the three DRA types at $0.05 \mathrm{wt} . \%$ in an $8 \mathrm{~mm}$ pipeline. The DR effect for the three types can be ranked as follows: cationic type $<$ anionic type $<$ nonionic type. Figure 19 shows the DR\% results for the three DRA types at $0.05 \%$ in a $3 \mathrm{~mm}$ fracture. In fractures, the DR effect can be ranked as follows: anionic type $<$ nonionic type $<$ cationic type. By comparing the results of electrical characteristics, cationic polymer shows the worst drag reduction performance in pipeline flow, in contrast to show the best effect in fracture flow. It indicated that the flow characteristics in the tortuous and rough fractures have an enhancement in the drag reduction effect of cationic polymers. The results may attribute to cationic polymer that has more active groups, which is more conducive to drag reduction effect under low flow rate.

3.3. DR Mechanism. Different from the calculation of flow drag in standard circular section pipelines, it is difficult to accurately calculate the internal flow drag of fractures with real tortuosity and roughness. Therefore, the visualization fracture model is used to observe the flow drag reduction characteristic in this study. Furthermore, the red tracer particles are added in the fluids, in order to get a more accurately description of the flow characteristic and analyse the drag reduction mechanism. The whole flow process in fractures can be divided into four stages, as shown in Figure 20.

(1) A circuitous surface can help the flow generate local vortexes as flow starts. Each recess has scale-matched vortexes that restrict the spanwise motions of turbulent vortexes, avoiding more perturbance as the surface is not regular, which increases the DR\%. At a low flow rate, the convex area can induce interior flow and facilitate formation of the DR structure, which increases the DR\%

(2) The scale-matched vortex balance is broken as the velocity increases; many vortexes in the concave area weaken the positive effect of scale-matched vortexes. However, the convex area is beneficial for the DR\% 


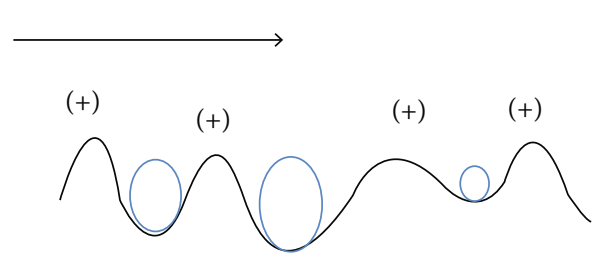

(1)

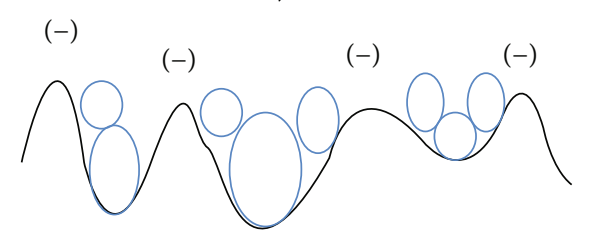

(3)

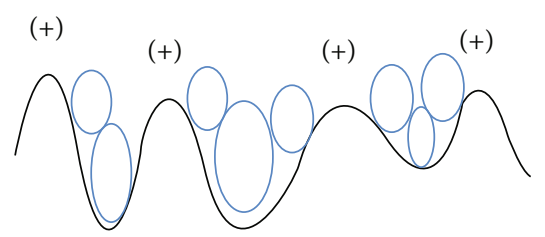

(2)

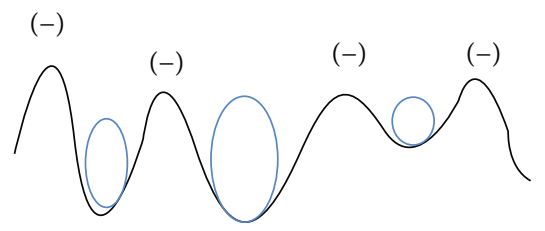

(4)

FIGURE 20: Images of DR mechanism.

(3) When the velocity reaches a critical value, the convex area tends to increase the shear force and facilitate formation of the DR structure, which helps increase turbulence and decrease the DR\%. As the flow rate increases, the negative influence gradually becomes apparent

(4) As the flow rate increases, many small vertexes result from the shear force caused by the convex area, and the larger vortexes disappear. The negative effect of many smaller or larger vortexes in the concave area starts to diminish, reaching a balance with their counterparts

The division of the flow stages indicates a clearer mechanism for the change rule of the drag reduction performance. At low flow rates, turbulence is concentrated mainly on the fracture surface, especially in the recessed areas. At this time, the DR effect of the drag reducer is not obvious; as the flow rate increases, the turbulence increases, and the polymer starts to play a role in both the recessed and raised areas, producing an increase in the DR rate. As the flow rate is further increased, the turbulence is concentrated near the centre of the flow channel. In this stage, the DR effect occurs mainly in the middle of the fractured flow channel, similar to pipeline flow, and gradually reaches its greatest effect. The flow stage characteristics further reveal the rules of DR performance in fracture flow with changes in the flow rate.

\section{Conclusions}

In this study, a self-developed indoor visualization drag testing cycle system considering fracture roughness and tortuosity was constructed to simultaneously test pipeline flow drag and fracture flow drag. Multiple experiments were conducted to determine the factors influencing the DR effect in fractures including concentration, temperature, molecular weight, and electrical characteristics. Pipeline flow tests were used as a reference to explore the drag reduction character- istic in fracture flow. Based on the experimental differences between pipelines and fractures, the main conclusions are as follows:

(1) For the pipeline flow, as the flow rate increases, the drag reduction effect will get better and eventually reach the optimal drag reduction rate. Then, if the flow rate further increases, the drag reduction rate will decrease (the highest pipeline flow rate in experiment is $16 \mathrm{~m} / \mathrm{s}$ ). When it comes to fracture flow, the flow rate has a similar influence on the drag reduction rate. However, limited by experimental conditions, the highest flow rate in the fracture is only $1.2 \mathrm{~m} / \mathrm{s}$. For both pipeline flow and fracture flow, they all have an optimal drag reduction rate. Before reaching this rate, the flow rate has a positive correlation with DR effect

(2) The experimental results of concentration influence show that, in pipeline flow, concentration has an adverse effect on the drag reduction performance under low flow rate conditions because high viscosity has a more significant negative impact on DR effect and leads to an increase in energy consumption. However, as the flow rate further increases, the polymer has sufficient space for expansion, and the fully stretched chain structure is more conducive to drag reduction performance. As for fracture flow, there is a critical drag reduction concentration. When the concentration is greater than $0.10 \mathrm{wt} . \%$, a negative DR effect begins to appear. The flow drag in fractures is rising with the growth in concentration of drag reducer

(3) The increase of temperature leads to the decrease of the viscosity of drag reducer solution, which is beneficial to the drag reduction effect under the low flow rate conditions. However, high temperature (greater than $55^{\circ} \mathrm{C}$ ) makes the DRA more vulnerable to wall shear influence and finally causes reduction in drag reduction effect under high flow rate conditions 
(4) In pipeline flow and fracture flow, the regular of drag reduction performance influenced by the molecular weight is opposite. Under low flow rate conditions, within a certain range of molecular weight increase, drag reduction effect is improved in pipeline flow but reduced in fracture flow. As for molecular structure, longer side groups can improve the form of structures to reserve more dissipated energy in the central of the pipeline flow. However, in fracture flow, excessive short side group is not conducive to the DR effect near the fracture surface

\section{Data Availability}

The experiment data used to support the findings of this study are available from the corresponding author upon request.

\section{Conflicts of Interest}

The authors declare no financial conflicts of interest regarding the publication of this paper.

\section{Authors' Contributions}

Professor Fujian Zhou, as the corresponding author of this paper, provided research ideas and funding. Professor Hongkui Ge provided guidance for experimental research methods and problems encountered in the experiment. Zhiyu Liu was the first author and responsible for experiment design and data analysis. Fan Fan as co-first author improved the experiments and added the test data. Donghang Zhang was the main experiment operator who finished the testing processes. Yang Li provided the test materials and was responsible for collecting and testing the parameters. Yuan Li and Kai Yang built and improved the experimental equipment and were responsible for drawing the flow chart of the experimental equipment. Fan Fan contributed equally to this work and co-first author.

\section{Acknowledgments}

This study is supported by the Shengli Oil Filed Luming Co., Ltd., "Research and Application of Temporary Plugging Technology in water fracture displacement of Beach-bar Sand Reservoir water fracture displacement" (LMKJ202105) and the Xinjiang Deli Zhongcheng Petroleum Engineering Co., Ltd., "Research on new drag reduction materials suitable for different fluid flow conditions" (ZC-20201209). The authors would like to acknowledge the financial support from the National Natural Science Foundation of China (No. 52174045).

\section{References}

[1] B. Yang, J. Z. Zhao, J. C. Mao, H. Tan, Y. Zhang, and Z. Song, "Review of friction reducers used in slickwater fracturing fluids for shale gas reservoirs," Journal of Natural Gas Science and Engineering, vol. 62, pp. 302-313, 2019.
[2] A. Muntasheri and G. A. Critical, "Review of hydraulic fracturing fluids over the last decade," in SPE Western North American and Rocky Mountain Joint Meeting, Denver, Colorado, 2014.

[3] R. Barati and J. Liang, "A review of fracturing fluid systems used for hydraulic fracturing of oil and gas wells," Journal of Applied Polymer Science, vol. 131, 2014.

[4] C. Robart, M. Ruegamer, and A. Yang, "Analysis of U.S. hydraulic fracturing design trends," in Paper Presented at the SPE Hydraulic Fracturing Technology Conference, The Woodlands, Texas, USA, 2013.

[5] H. D. Brannon and C. E. Bell, "Eliminating slickwater fracturing compromises for improved shale stimulation," in SPE Annual Technical Conference and Exhibition, Denver, Colorado, USA, 2011.

[6] G. Y. Gao, C. H. Wang, H. Zhou, and P. Wang, "Modified fracture mechanics approach for hydraulic fracturing stress measurements," Geofluids, vol. 2020, Article ID 8860163, 11 pages, 2020.

[7] Z. P. Sun, H. L. Zhang, Z. F. Wei et al., "Effects of slick water fracturing fluid on pore structure and adsorption characteristics of shale reservoir rocks," Journal of Natural Gas Science and Engineering, vol. 51, no. 2, pp. 27-36, 2018.

[8] H. Belyadi, E. Fathi, and F. Belyadi, "Chapter Five - Hydraulic Fracturing Fluid Systems," in Hydraulic Fracturing in Unconventional Reservoirs (Second Edition), pp. 47-69, Gulf Professional Publishing, 2019.

[9] B. A. Toms, "Some observations on the flow of linear polymer solutions through straight pipelines at large Reynolds numbers," in Proceedings of the 1st International Congress on Rheology, pp. 135-141, Amsterdam, 1948.

[10] G. Sitaramaiah and C. L. Smith, "Turbulent drag reduction by polyacrylamide and other polymers," Society of Petroleum Engineers Journal, vol. 9, no. 2, pp. 183-188, 1969.

[11] Y. H. Li, G. R. Chesnut, R. D. Richmond, G. L. Beer, and V. P. Caldarera, "Laboratory tests and field implementation of gasdrag-reduction chemicals," SPE production \& facilities, vol. 13, pp. 53-58, 1998.

[12] W. Brostow, "Drag reduction in flow: review of applications, mechanism and prediction," Industrial and Engineering Chemistry, vol. 14, no. 1, pp. 409-416, 2008.

[13] W. W. Li and R. K. Saini, "Polyacrylamide grafted polysaccharide as friction reducer for slickwater fracturing treatment," Journal of Macromolecular Science, Part A, vol. 58, no. 4, pp. 243-248, 2021.

[14] M. L. Luo, X. D. Si, M. Z. Li, X. H. Jia, Y. L. Yang, and Y. P. Zhan, "Experimental study on the drag reduction performance of clear fracturing fluid using wormlike surfactant micelles and magnetic nanoparticles under a magnetic field," Nanomaterials, vol. 11, no. 4, p. 885, 2021.

[15] Y. F. Zhang, F. B. Zhou, and J. H. Kang, "Flow and heat transfer in drag-reducing polymer solution flow through the corrugated tube and circular tube," Applied Thermal Engineering, vol. 174, pp. 115185-115185, 2020.

[16] N. le Brun, I. Zadrazil, L. Norman, A. Bismarck, and C. N. Markides, "On the drag reduction effect and shear stability of improved acrylamide copolymers for enhanced hydraulic fracturing," Chemical Engineering Science, vol. 146, pp. 135-143, 2016.

[17] J. GUO, Y. LI, and S. WANG, "Adsorption damage and control measures of slick-water fracturing fluid in shale 
reservoirs," Petroleum Exploration and Development, vol. 45, no. 2, pp. 336-342, 2018.

[18] Z. Y. Liu, F. J. Zhou, H. Y. Qu, Z. Yang, Y. S. Zou, and D. B. Wang, "Impact of the microstructure of polymer drag reducer on slick-water fracturing," Geofluids, vol. 2017, Article ID 9080325, 8 pages, 2017.

[19] A. Abubakar, T. al-Wahaibi, Y. al-Wahaibi, A. R. al-Hashmi, and A. al-Ajmi, "Roles of drag reducing polymers in singleand multi-phase flows," Chemical Engineering Research and Design, vol. 92, no. 11, pp. 2153-2181, 2014.

[20] Z. Lufeng, Z. Fujian, Z. Shicheng, L. zhun, W. Jin, and W. Yuechun, "Evaluation of permeability damage caused by drilling and fracturing fluids in tight low permeability sandstone reservoirs," Journal of Petroleum Science and Engineering, vol. 175, pp. 1122-1135, 2019.

[21] D. Wang, S. Zlotnik, P. Díez, H. Ge, F. Zhou, and B. Yu, “A numerical study on hydraulic fracturing problems via the proper generalized decomposition method," Computer Modeling in Engineering and Sciences, vol. 122, no. 2, pp. 703-720, 2020.

[22] D. Wang, Y. Dong, D. Sun, and B. Yu, “A three-dimensional numerical study of hydraulic fracturing with degradable diverting materials via CZM-based FEM," Engineering Fracture Mechanics, vol. 237, p. 107251, 2020.

[23] X. Bo, Z. Shicheng, L. Xin, Z. Tong, Z. Bo, and C. Laijun, "Optimization and evaluation of drag reducer of slickwater fracturing in shale gas reservoir," Oil and gas geology and recovery, vol. 21, no. 2, pp. 102-105, 2014.

[24] K. Zhang, H. J. Choi, and C. H. Jang, "Turbulent drag reduction characteristics of poly (acrylamide-co-acrylic acid) in a rotating disk apparatus," Colloid and Polymer Science, vol. 289, no. 17-18, pp. 1821-1827, 2011.

[25] Y. M. Cheng, "Impact of water dynamics in fractures on the performance of hydraulically fractured wells in gas-shale reservoirs," Journal of Canadian Petroleum Technology, vol. 51, no. 2, pp. 143-151, 2012.

[26] N. Liu, Z. Zhang, Y. Zou, X. Ma, and Y. Zhang, "Propagation law of hydraulic fractures during multi-staged horizontal well fracturing in a tight reservoir," Petroleum Exploration and Development, vol. 45, no. 6, pp. 1129-1138, 2018.

[27] Y. P. Sun, Q. H. Wu, M. Z. Wei, B. Bai, and Y. Ma, "Experimental study of friction reducer flows in microfracture," Fuel, vol. 131, pp. 28-35, 2014.

[28] Z. J. Song, L. B. Liu, J. R. Hou, B. Bai, and W. Su, "Effect of polymer on gas flow behavior in microfractures of unconventional gas reservoirs," Journal of Natural Gas Science and Engineering, vol. 23, pp. 26-32, 2015.

[29] F. C. Li, Y. Kawaguchi, B. Yu, J. J. Wei, and K. Hishida, "Experimental study of drag-reduction mechanism for a dilute surfactant solution flow," International Journal of Heat and Mass Transfer, vol. 51, no. 3-4, pp. 835-843, 2008.

[30] F. Fan, F. Zhou, and Z. Liu, "Experimental and Numerical Study on Drag Reduction Performance of Slickwater in Turbulent Pipeflow," in International Field Exploration and Development Conference, pp. 471-484, Springer, 2018.

[31] J. Fink, "Chapter 12 - Drag Reducers," in Petroleum Engineer's Guide to Oil Field Chemicals and Fluids (Third Edition), pp. 517-529, Gulf Professional Publishing, 2021.

[32] C. Zou, D. Dong, Y. Wang et al., "Shale gas in China: characteristics, challenges and prospects (II)," Petroleum Exploration and Development, vol. 43, no. 2, pp. 182-196, 2016. 